\title{
International survey of orthokeratology contact lens fitting
}

DOI:

10.1016/j.clae.2018.11.005

\section{Document Version}

Accepted author manuscript

Link to publication record in Manchester Research Explorer

\section{Citation for published version (APA):}

Morgan, P. B., Efron, N., Woods, C. A., \& Santodomingo-Rubido, J. (2019). International survey of orthokeratology contact lens fitting. Contact Lens and Anterior Eye. https://doi.org/10.1016/j.clae.2018.11.005

\section{Published in:}

Contact Lens and Anterior Eye

\section{Citing this paper}

Please note that where the full-text provided on Manchester Research Explorer is the Author Accepted Manuscript or Proof version this may differ from the final Published version. If citing, it is advised that you check and use the publisher's definitive version.

\section{General rights}

Copyright and moral rights for the publications made accessible in the Research Explorer are retained by the authors and/or other copyright owners and it is a condition of accessing publications that users recognise and abide by the legal requirements associated with these rights.

\section{Takedown policy}

If you believe that this document breaches copyright please refer to the University of Manchester's Takedown Procedures [http://man.ac.uk/04Y6Bo] or contact uml.scholarlycommunications@manchester.ac.uk providing relevant details, so we can investigate your claim.

\section{OPEN ACCESS}




\title{
Manuscript Details
}

\section{Manuscript number}

Title

Article type
CLAE_2018_261_R1

International survey of orthokeratology contact lens fitting

\begin{abstract}
Purpose. To determine the extent of orthokeratology (OK) contact lens fitting worldwide and to characterize associated demographics and fitting patterns. Methods. Survey forms were sent to contact lens fitters in 45 countries between January and March every year for 14 consecutive years (2004 - 2017, inclusive). Practitioners were asked to record data relating to the first 10 contact lens fits or refits performed after receiving the survey form. Results. Data were obtained for 295,044 contact lens fits, of which 2,702 were with OK lenses and 292,342 were with other lens types (non-OK). Overall, OK lenses represented $1.2 \%$ of all contact lens fits, with significant differences between nations ( $p$ $<0.0001$ ), ranging from no fits recorded in Brazil, Egypt, Indonesia, Iran, Lithuania, Nepal and the United Arab Emirates, to $6.0 \%$ in The Netherlands. There has been a slight overall increase in OK lens fitting over the survey period $(p<0.0001)$. OK lenses were fitted to younger persons (OK, $25.0 \pm 12.8$ years vs. non-OK, $39.8 \pm 14.9$ years) $(p<0.0001)$. A higher proportion of males $(55 \%)$ were fitted with OK lenses versus non-OK lenses $(30 \%)(p<0.0009)$. There was a skewed distribution towards OK lenses being fit with higher oxygen permeable materials $(p<0.0001)$ and on a planned replacement basis $(p<0.0001)$. Conclusions. OK contact lens prescribing is a niche activity, with this lens type typically being fitted in high oxygen permeable materials on a planned replacement basis to younger males. The slightly increasing rate of OK fitting, albeit at a very low level, may be attributed to practitioner interest in the reported myopia control properties of this lens type.
\end{abstract}

\section{Keywords}

Corresponding Author

Order of Authors contact lenses; fitting; orthokeratology; survey

Nathan Efron

Philip Morgan, Nathan Efron, Craig Woods, Jacinto Santodomingo-Rubido

\section{Submission Files Included in this PDF}

\section{File Name [File Type]}

Response to Reviewers.docx [Response to Reviewers (without Author Details)]

Highlights.doc [Highlights]

Title Page.doc [Title Page (with Author Details)]

Manuscript.doc [Manuscript (without Author Details)]

Figure 1.pdf [Figure]

Figure 2.jpeg [Figure]

Figure 3.pdf [Figure]

Figure 4.pdf [Figure]

To view all the submission files, including those not included in the PDF, click on the manuscript title on your EVISE Homepage, then click 'Download zip file'.

\section{Research Data Related to this Submission}

There are no linked research data sets for this submission. The following reason is given:

Data will be made available on request 


\section{Response to Reviewers \\ International survey of orthokeratology contact lens fitting}

Reviewers comments in bold font

Our response in plain font

Comments from the editors and reviewers:

Authors should note that the Guide for Authors for CLAE states 'Authors are discouraged from the use of personal pronouns in the course of their writing.' Please carefully check the spelling and grammar of the paper and if necessary, have this checked by a language editor. Many authors mix up different writing styles, however at CLAE the 'third person scientific' style is considered appropriate and is preferred. Please do not use personal pronouns such as 'our' or 'we'. For example, instead of 'our study....', or 'we found..', authors should state 'this study....'or 'it was found...'.

We have removed all personal pronouns from the manuscript.

Please ensure you upload a document that contains the reviewers' comments and your answers to these comments so that it is easy to see where you have addressed the comments in the revised version.

This is that document.

Can you ensure that you answer the criticisms of the second reviewer please? We have responded to all comments of both reviewers.

One suggested change not listed below is the use of certain phrases.

We have amended our phraseology is response to these suggestions.

Both reviewers have commented on the lack of supporting refs. References have been added as per the suggestions of the referees.

"The authors seem to have no confidence in the use of OK to control myopia progression. Their continued use of terms such as 'purported' border on insulting to the large number of researchers who have published studies which have shown a strong positive effect.

It was never out intention to insult orthokeratology researchers. We have replaced the word 'purported' with 'reported'.

As such I feel that the paper should not be published without including reference to these studies and removing these disparaging comments. References have been included and we have replaced the word 'purported' with 'reported'.

I accept there is a small increase in risk of MK if strict adherence to care routine is not implemented, but this does not mean the treatment is ineffective. 
We have not suggested anywhere in the paper that the treatment is ineffective.

As a survey alone, yes it provides a lot of data from a very large sample of optometrists but the study aimed to address OK use. It should have addressed prescriptions to children and young adults and asked why practitioners that had made this choice of intervention.

This reviewer seems to be suggesting that we should have conducted a different study - presumably about OK fitting for myopia control. However, this was not our intention. We were interested in documenting the extent and demographics of all OK fitting. It is likely that most practitioners fit OK lenses for the classical benefit of not having to wear any optical correction during the waking hours. As such, these lenses are fitted to persons of all ages, not just children and young adults. This fact is borne out of the observation in this study that about $60 \%$ of OK lenses are being fitted to adults (see Figure 4). ! It would appear, as this reviewer is suggesting, that some practitioners fit OK lenses exclusively for myopia control, but this is likely to be the minority of fits. So, to be clear, this paper is about ALL OK fitting, not just OK fitting for myopia control. Our data analysis, statistical analysis, presentation of results and discussion are consistent with the stated aim of this work as stated in the Abstract: "To determine the extent of orthokeratology (OK) contact lens fitting worldwide and to characterize the associated demographics and fitting patterns."

It almost appears to have the intention of discrediting OK by indicating it is a rarely used treatment of dubious value."

The reviewer perhaps wishes that $90 \%$ of all contact lens fits are OK, but the reality is, based on our survey of 295,044 contact lens fits worldwide, that OK is a rarely used treatment, representing just over $1 \%$ of all contact lens fits. Again, we have not stated in this paper that $\mathrm{OK}$ is of dubious value, and in response to the suggestion of this reviewer, we have altered our use of language to avoid such misinterpretation.

\section{Reviewer 1}

Review paper on use of OK globally. Mainly there should be more clinical references in the paper to support your statements as to why you have the results.

This paper is about OK fitting, not myopia control, as explained above. Nevertheless, we have now included additional papers that demonstrate the efficacy of OK for myopia control.

Line 43 regresses to its former shape would only happen if OK is only for one night or OK is discontinued after a few weeks...not sure what you want to say here...that there is on a daily basis regression of 0.50 to $0.75 \mathrm{D}$.... please clarify. Figure 4 of Nichols et al (Ref 3] clearly shows that the eye continues to become more myopic throughout the waking hours, for up to 60 days after the commencement of OK. However, to avoid confusion, we have deleted the phrase "... during which the cornea regresses towards its former shape."

\section{Line $\mathbf{4 5}$ Is disbelief a word? use risks}

The word we used was 'disbenefit', not 'disbelief'. 'Disbenefit' is defined by the Living Oxford English Dictionary (on-line) as: "A disadvantage or loss resulting from something". This is precisely our intended use. We are not referring to risks. 
Line 51 only 1 reference regarding MC use...a review paper....perhaps better to reference the papers that the review paper references instead as it sounds that OK is not successful for MC.

This paper is about OK fitting, not myopia control. Nevertheless, we have now added 15 papers (references 5-19) that demonstrate the efficacy of OK for myopia control.

Line 188 reference again only to global trends but no clinically related references are used....please add.

The discussion here relates to practitioner perceptions, not to the question of the efficacy of myopia control. The latter point has been addressed by the addition of 15 new references (references 5-19).

Line 190-2 "perceived ability to arrest Myopia progression" change arrest to slow and this should be referenced as above.

We have now used the word 'slow'. As stated above, we have now added 15 papers (references 5-19) that demonstrate the efficacy of OK for myopia control.

Line 207 replacement due to altering lens design (reference this) or could be due to the state of the GP lens surface (scratches and deposits) with ON wear (please reference this).

We have added a reference in relation to the requirement to replace lenses to effect a change in lens design. We could find no references to support the notion that GP lenses develop more scratches and deposits when worn overnight.

\section{Reviewer 2}

This is a large scale study which claims to determine the extent of OK fitting worldwide. However, the study design which investigated CL fitting in general fails to address several important issues.

Yes, it is indeed large scale study. We have addressed these important issues, as described below.

1. The study targeted patients of all ages, rather than the most likely patients, children and adolescents. The survey should have addressed the first 10 patients in this age group.

As mentioned above, our aim was to survey OK fitting, not myopia control. We sought to discover the extent of $\mathrm{OK}$ fitting to all age groups across numerous countries. Our stated aim is clear and unambiguous. We are unable to respond to this apparent criticism that we have conducted the wrong study.

2. There was no question regarding the choice of treatment - why did a practitioner prescribe OK or another CL modality, especially for children. We agree that it could have been interesting to know why practitioners fit different lens types. However, this paper extracts data from a survey that has been conducted in up to 60 countries for almost a quarter of a century. Our survey does not ask why certain lenses are fitted. Thus, we cannot answer this question. 
The authors seem to have little or no belief in the effectiveness of OK in controlling myopia progression as evidenced in several paragraphs in the manuscript. This despite evidence from both individual studies and metaanalyses. This does make one wonder why the authors undertook such a large scale study to investigate an intervention they considered of little value. This paper is not about OK for myopia control. It is about the demographics of OK fitting for whatever reason. We have not stated anywhere in the paper that OK "is of little value".

\section{Specific Points}

\section{Introduction}

Line 45. Would prefer disadvantages to disbenefits.

'Disbenefit' is defined by the Living Oxford English Dictionary (on-line) as: "A disadvantage or loss resulting from something". 'Disadvantage' is defined by the Living Oxford English Dictionary (on-line) as: "an unfavourable circumstance or condition that reduces the chances of success or effectiveness." Our use of the work 'disbenefit' is precisely as intended. We are not referring to disadvantages, as defined above.

Line 47-51. and Lines 178 -180. The effectiveness of OK for myopia control has been shown by a number of trials, including RCCT, in several countries. Reduction in axial growth and reduction in amount of myopia has been shown to be highly significant. Several meta-analyses have confirmed individual results. I feel your wording here is somewhat disparaging of the effectiveness of the treatment and would suggest a rather more positive wording.

As discussed above, we have altered the wording to be more positive, and have now added 15 references that support the efficacy of OK for myopia control.

\section{Methodology}

Line 89 , As OK is a specialised treatment and would not be expected to be useful for most clients, I wonder why only 10 fits of CL were recorded. As the major beneficiaries of OK are children, would not the treatment offered to the first 10 myopic children be more useful?

Our aim was to survey OK fitting, not myopia control. We sought to discover the extent of OK fitting to all age groups across numerous countries. Our stated aim is clear and unambiguous. We are unable to respond to this apparent criticism that we have conducted the wrong study.

\section{Results}

Line 141-144. This analysis of age groups does help to show use of OK in children and adolescents referred to above, though I still believe concentration on these age groups would have been more useful.

We sought to discover the extent of OK fitting to all age groups across numerous countries. Our stated aim is clear and unambiguous. We are unable to respond to this apparent criticism that we have conducted the wrong study. 
Line 146. Was any reason proposed for the skewing of male:female ratio?

The reason for the skewing of male:female ratio is unclear. We have stated this. It is not unusual in published research that some of the findings do not have an apparent explanation, and this is one of those instances.

Line 152-3 I was surprised to read that materials with low or even zero DK were still in use and assume these were in low income countries.

The reviewer is possibly correct in this assumption, however, the number of fits with materials with low or even zero DK was too low to enable a meaningful statistical analysis of the relation between the fitting of such lenses and country GDP.

Line 160 . I would suggest the word practical is replaced by clinical We have replaced the word 'practical' with 'clinical'.

\section{Discussion}

Lines 178-180. Please see my comments above. The use of the word purported here is not appropriate. The term indicates false claims or those not easy to believe. This almost accuses a large number of well-respected optometrists of falsifying data and must be corrected.

We have replaced the word 'purported' with 'reported'.

Lines 187-188. This is certainly the experience in HK and China, where there is increasing popularity of OK fitting. Why would there be growing appreciation in practitioners if the practice is not effective?

The growing appreciation may relate more to practitioner perceptions, beliefs and hopes, hence the deliberate use of the term "appreciation of practitioners". To demonstrate that the practice is effective, we have now included 15 references in the paper (references 5-19) that prove the efficacy OK fitting for myopia control.

Lines $190-195$, Unfortunately here again is a return to scepticism of the usefulness of the technique with use of the word "Perceived' Clearly if the main reason for choice of OK is myopia control, younger patients would be the target of the treatment. One would generally not offer the treatment to those who are unlikely to experience deterioration of their myopia.

The reviewer seems to be confusing the concepts of perception and reality. In reality, myopia control with OK lenses does work, as evidenced by the 15 new papers we have now included in the paper (references 5-19). However, this section seeks to explain fitting trends in terms of the perceptions of practitioners. These are two very different issues.

Lines 207-8. While fitting may need adjustment, replacement also needed to provide new lenses for those inadvertently damaged during Cleaning and handling.

While it is true that more regular lens replacement is beneficial to avoid excess lens scratching and deposition, we believe that the very large difference between OK and non-OK replacement rates is better explained by the requirement to continuously modify the lens fit to maintain the corneal moulding effect. 


\section{Conclusion}

The wording here addresses one of the major inadequacies of this study. The authors state presumably for arresting myopia progression If the purpose of the study was to assess the use of OK, surely they should have determined why practitioners were using this intervention.

This issue is addressed above. 


\section{Highlights}

- A survey of orthokeratology prescribing was conducted annually in 45 countries between 2004 and 2017, inclusive.

- Orthokeratology fits represented $1.2 \%$ of all contact lens fits, with significant differences between nations.

- A higher proportion of males (55\%) were fitted with orthokeratology lenses versus nonorthokeratology lenses $(30 \%)$.

- There was a skewed distribution towards orthokeratology lenses being fit with higher oxygen permeable materials and on a planned replacement basis. 


\section{International survey of orthokeratology contact lens fitting}

Philip B. Morgan ${ }^{\text {a }}$, Nathan Efron ${ }^{\mathrm{b} *}$, Craig A. Woods ${ }^{\mathrm{c}}$, Jacinto Santodomingo-Rubido ${ }^{\mathrm{d}}$, and The International Contact Lens Prescribing Survey Consortium

${ }^{a}$ Eurolens Research, Division of Pharmacy and Optometry, The University of Manchester, UK binstitute of Health and Biomedical Innovation, and School of Optometry and Vision Science, Queensland University of Technology, Australia

${ }^{\mathrm{c} S c h o o l}$ of Medicine (Optometry), Deakin University, Australia

${ }^{\mathrm{d} M e n i c o n}$ Co., Ltd., Nagoya, Japan

*Corresponding author at:

Institute of Health and Biomedical Innovation, and School of Optometry, Queensland University of Technology, Kelvin Grove, Queensland 4059, Australia.

E-mail address: n.efron@qut.edu.au (N. Efron)

4 Figures, 0 Tables

Running header: Orthokeratology fitting survey 


\section{International survey of orthokeratology contact lens fitting}

Philip B. Morgan ${ }^{\text {a }}$, Nathan Efron ${ }^{\mathrm{b} *}$, Craig A. Woods ${ }^{\mathrm{c}}$, Jacinto Santodomingo-Rubido ${ }^{\mathrm{d}}$, and The International Contact Lens Prescribing Survey Consortium

${ }^{a}$ Eurolens Research, Division of Pharmacy and Optometry, The University of Manchester, UK binstitute of Health and Biomedical Innovation, and School of Optometry and Vision Science, Queensland University of Technology, Australia

${ }^{\mathrm{c} S c h o o l}$ of Medicine (Optometry), Deakin University, Australia

${ }^{\mathrm{d} M e n i c o n}$ Co., Ltd., Nagoya, Japan

*Corresponding author at:

Institute of Health and Biomedical Innovation, and School of Optometry, Queensland University of Technology, Kelvin Grove, Queensland 4059, Australia.

E-mail address: n.efron@qut.edu.au (N. Efron)

4 Figures, 0 Tables

Running header: Orthokeratology fitting survey 


\section{ABSTRACT}

Purpose. To determine the extent of orthokeratology (OK) contact lens fitting worldwide and to characterize associated demographics and fitting patterns.

Methods. Survey forms were sent to contact lens fitters in 45 countries between January and March every year for 14 consecutive years (2004 - 2017, inclusive). Practitioners were asked to record data relating to the first 10 contact lens fits or refits performed after receiving the survey form. Results. Data were obtained for 295,044 contact lens fits, of which 2,702 were with OK lenses and 292,342 were with other lens types (non-OK). Overall, OK lenses represented $1.2 \%$ of all contact lens fits, with significant differences between nations $(\mathrm{p}<0.0001)$, ranging from no fits recorded in Brazil, Egypt, Indonesia, Iran, Lithuania, Nepal and the United Arab Emirates, to $6.0 \%$ in The Netherlands. There has been a slight overall increase in OK lens fitting over the survey period $(\mathrm{p}<$ 0.0001). OK lenses were fitted to younger persons (OK, $25.0 \pm 12.8$ years vs. non-OK, $39.8 \pm 14.9$ years) $(\mathrm{p}<0.0001)$. A higher proportion of males $(55 \%)$ were fitted with OK lenses versus non-OK lenses $(30 \%)(\mathrm{p}<0.0009)$. There was a skewed distribution towards OK lenses being fit with higher oxygen permeable materials $(\mathrm{p}<0.0001)$ and on a planned replacement basis $(\mathrm{p}<0.0001)$.

Conclusions. OK contact lens prescribing is a niche activity, with this lens type typically being fitted in high oxygen permeable materials on a planned replacement basis to younger males. The slightly increasing rate of OK fitting, albeit at a very low level, may be attributed to practitioner interest in the reported myopia control properties of this lens type.

Key words: contact lenses, fitting, orthokeratology, survey. 


\section{Introduction}

Orthokeratology (OK) has been discussed in the refereed literature for over half a century, with PubMed search for the term 'orthokeratology' revealing the first paper on the subject to have been published by Ziff in 1968 [1]. In its original form, OK involved wearing flat-fitting hard lenses made from polymethyl methacrylate (PMMA) during the waking hours, with the intention of progressively flattening the cornea to reduce the degree of myopia, so as to afford reasonable vision following lens removal [2].

The introduction of highly gas-permeable rigid materials, reverse geometry designs and instrumentation to monitor changes in corneal topography has allowed the correction of low to moderate levels of myopia and astigmatism with OK lenses, shifting the approach from daytime wear of lenses to overnight wear (so-called 'overnight orthokeratology' [3]). Contemporary OK lenses are worn overnight to induce a flattening of central corneal curvature to temporarily correct myopia, and are removed upon waking to provide reasonable vision throughout most of the waking hours [3]. Practitioners fitting such lenses are obliged to weigh up the benefits of this approach (minimizing lens discomfort due to closed-eye lens wear during sleep) versus the disbenefits (increased risk of microbial keratitis during overnight lens wear [4]).

More recently, it has been proposed that $\mathrm{OK}$ has an additional benefit of arresting the progression of myopia (so-called 'myopia control') [5-19]. This is thought to occur as a result of the optical configuration of reverse geometry lenses and its impact on peripheral corneal molding, whereby positive spherical aberration in the molded corneal periphery creates an apparent reduction in the optical stimulus for eye growth [20].

Although there has been much discussion about $\mathrm{OK}$ in the referred literature, clinical magazines, internet forums, conferences and the like, little is known of the true extent to which these lenses are fitted around the world. This paper examines trends in, and factors associated with, OK contact lens fitting in 45 countries over a 14 year period (2004-2017, inclusive). Such information can provide (a) a valuable yardstick for contact lens clinicians, against which they can assess their own prescribing approaches to $\mathrm{OK}$, and (b) useful guidance to the contact lens industry on the clinical utilization of this specialist lens category. 


\section{Materials and methods}

\subsection{Conduct of the annual survey}

Between January and March each year from 2004 to 2017, a contact lens fitting survey was distributed in 45 countries. This was achieved through the offices of members of the International Contact Lens Prescribing Survey Consortium (see Acknowledgements), which is a network of academics, industry representatives, and clinical colleagues who have agreed to manage the survey in their country or geographic region, as outlined below.

Each Consortium member was requested to send a paper or electronic (e-mail) survey form to as many contact lens practitioners (opticians, optometrists and/or ophthalmologists, depending on the market) in their country as possible, with a notional target of 1,000 surveys sent. Some smaller countries were unable to reach this target due to the limited number of practitioners in that region.

In some countries, such as Australia and Spain, survey forms were sent to virtually every practitioner in the country (about 5,000 and 6,000 practitioners, respectively). In other countries, survey forms were sent to a selection of practitioners throughout the country; this process varied among countries, ranging for example from a computer-generated random list of exactly 1,000 recipients from the national practitioner register in the UK, to a list of practitioner-subscribers to contact lens trade publications in the USA and Russia. In large countries with dispersed populations, such as China and Russia, survey forms were sent to practitioners in a select number of major urban cities. The survey forms were sent together with a request that they be completed and returned within three months of receipt. To minimize any possible seasonal influences or potential impact of the annual business activity cycle on the types of lenses fit, the survey was conducted in the first few months of each year in all countries.

The same survey format was used each year, as published previously [21]. This standardized, onesided survey form was locally translated. The form guided practitioners to enter a number of background details and to supply generic information about the first ten contact lens fits performed after receipt of the survey form. For each contact lens fitting, practitioners were requested to complete the following details: date of fitting, new fitting or refitting, age and sex of patient, lens material, lens design, frequency of lens replacement, times per week of lens wear, wearing modality (daily or extended wear) and care system [21]. Practitioners were asked to return the form irrespective of the number of patients seen (if fewer than 10). The returned forms were logged and data from each form were manually entered into an Excel spread sheet (Microsoft Corporation, Redmond WA) to facilitate data analysis. 
The Office of Research Ethics at the University of Waterloo, Canada, granted approval for this work, and noted that, as the data being collected were (1) part of normal practice care provision, (2) transcribed from the practice patient records, and (3) de-identified in respect of the patients and source practice, then the requirement for patient informed consent was waived.

\subsection{Statistical analysis}

Differences among practitioners in relation to the amount of contact lens fitting performed were accounted for by assigning an appropriate weighting to each recorded contact lens fit. This was achieved by estimating the number of contact lens fits performed each year by each respondent (based on the date information provided on the survey form) and using this as a weighting factor. For example, the data generated by a practitioner completing all 10 fits in one week were given twice the weighting of a practitioner who fitted 10 patients in two weeks. Data were mined with the aid of the Excel ${ }^{\circledR}$ Pivot Table function. Analysis of the main differences within the dataset was conducted using logistic regression models (JMP, SAS Institute Inc., Cary NC) for OK lens fits. More detailed analyses - such as comparison of materials fitted and replacement intervals prescribed - were assessed using an unpaired Wilcoxon test.

\section{Results}

\subsection{Demographics}

Data were accessed from 45 countries, each reporting information about at least 500 contact lens fits, during the 14 year survey period (2004-2017). This generated a database of 295,044 contact lens fits, of which 2,702 were with OK lenses and 292,342 were with other lens types (non-OK). The median country response rate was 3,197 fits over this period, ranging from 508 fits in Egypt to 61,731 fits in Japan.

OK lenses represented $1.2 \%$ of all weighted contact lens fits; however, there was considerable variance in OK lens fitting among the 45 countries surveyed $\left(\chi^{2}=1,120, p<0.0001\right)$, ranging from no fits recorded in Brazil, Egypt, Indonesia, Iran, Lithuania, Nepal and the United Arab Emirates, to $6.0 \%$ in The Netherlands (Fig. 1). OK fits represented $1.4 \%$ of all contact lens fits in East and Southeast Asian nations ( $\mathrm{N}=9$ nations: China, Hong Kong, Japan, Indonesia, Korea, Malaysia, Philippines, Singapore and Taiwan) and 1.1\% of all contact lens fits in all other nations surveyed (N $=36$ nations); this difference was not statistically significant $\left(\chi^{2}=0.0, p=1.0\right)$. Fig. 2 is a colourcoded 'heat map', showing the extent of OK fitting around the world. 
The overall extent of OK contact lens fitting has slowly risen through the 14 year survey period $\left(\chi^{2}\right.$ $=266, \mathrm{p}<0.0001$ ), increasing from $0.5 \%$ of all lens fits in 2004 to $1.3 \%$ in 2017 (Fig. 3).

The mean age ( \pm standard deviation) of those fitted with OK lenses $(25.0 \pm 12.8$ years $)$ was lower than for non-OK fits $(39.8 \pm 14.9$ years $)\left(\chi^{2}=1,054, \mathrm{p}<0.0001\right)$.

Using an approach which the authors have described previously [22], data relating to individual lens wearers were stratified into groups that were considered to represent different stages of maturity, in respect of a combination of factors such as the prevalence of myopia, communication skills, lens handling capabilities and level of financial and contact lens-related decision-making independence. These age groups comprised infants ( $\leq 5$ years), children $(6-12$ years), teenagers $(13-17$ years) and adults ( $\geq 18$ years). The distribution of OK and non-OK lens fits in respect of these four age groups is presented in Fig. 4, which highlights a significantly skewed distribution towards OK lenses being fitted to younger age groups. As expected, there were no reported OK fits to infants.

A higher proportion of males (55\%) were fitted with OK lenses versus non-OK lenses $(30 \%)\left(\chi^{2}=\right.$ $11, \mathrm{p}<0.0009)$.

\subsection{Lens materials}

Rigid lens materials were categorized according to oxygen permeability (Dk), as zero Dk (polymethyl methacrylate), low-Dk $(<40)$, mid-Dk $(40-90)$ and high-Dk $(>90)$. The proportion of rigid lens fits in respect of this categorization was: zero Dk (polymethyl methacrylate) - OK 2\%, Non-OK 2\%; low-Dk - OK 1\%, Non-OK 13\%; mid-Dk - OK 5\%, Non-OK 40\%; and high Dk OK $92 \%$, Non-OK $45 \%\left(\chi^{2}=1447, \mathrm{p}<0.0001\right)$. These data indicate a skewed distribution towards OK lenses being fit with higher Dk materials.

\subsection{Lens use}

Lenses fitted for OK were worn $6.9 \pm 0.6$ days per week. Those fitted with other lens types (nonOK) wore lenses $6.7 \pm 0.9$ times per week. Although this difference was statistically significant $\left(\chi^{2}\right.$ $=49, \mathrm{p}<0.0001)$, it is not thought to be of any clinical importance.

The frequency of lens replacement was found to be as follows: monthly - OK 0\%, non-OK 4\%; 3-6 monthly - OK 3\%, non-OK 2\%; annually - OK 68\%, non-OK 36\%; and unplanned - OK 29\%, non-OK 58\% $\left(\chi^{2}=730, \mathrm{p}<0.0001\right)$. This suggests a higher proportion of OK lenses were fitted on a planned replacement basis. 


\section{Discussion}

Differences in the extent of OK lens prescribing between nations may be attributed to international differences in the training, attitudes and collective confidence of the predominant practitioner groups - opticians, optometrists and ophthalmologists. For example, ophthalmologists, who largely or exclusively manage serious contact lens-related eye infections in many regions of the world, may harbour more conservative attitudes and concerns relating to the ocular health risks of overnight OK lens wear.

The high proportion of $\mathrm{OK}$ lens prescribing in The Netherlands (6.0\%) may relate to a longstanding enthusiasm for rigid lens fitting in this country, fuelled by highly visible opinion leaders advocating this approach, as well as ready access to local specialized rigid lens laboratories who can custom design the required lenses [23]. Relatively high rates of OK fitting in Hong Kong $(5.9 \%)$ and Taiwan (3.6\%) could be related to the reported myopia control capabilities of OK, as a strategy to constrain the myopia epidemic in these countries; [24] however, an elevated rate of OK fitting was not evident across all of the East and Southeast Asian nations surveyed.

OK only represents $1.2 \%$ of all contact lens fits and must therefore be considered as a niche or 'specialist' approach to contact lens fitting. The small rise in OK fitting over the 14 year time span of this survey possibly reflects (a) an increasing interest in OK lens fitting among practitioners, (b) a growing awareness among lens wearers of $\mathrm{OK}$ as an alternative to conventional lens wear, and (c) a greater appreciation among practitioners and lens wearers of the potential for these lenses to reduce the rate of myopia progression [25].

The much younger age at which people are fitted with OK lenses is consistent with the perceived ability of this modality of lens wear to slow the progression of myopia during the critical stages of myopia development. Given that the active years for myopia development from an ocular growth perspective relate to those in the 'children' and 'teenager' age groups as defined in this study [26], it is not surprising that a disproportionately greater number of $\mathrm{OK}$ fits were recorded for these groups. The reason for the higher proportion of $\mathrm{OK}$ fits among males is unclear.

There is a school of thought that contends OK lenses may not need to be worn every night of the week to maintain their efficacy; however, data from this survey suggests OK lenses are being worn every night to maintain the daily corneal moulding effect required to improve vision [3]. The high 
frequency of use of non-OK lenses (predominantly soft lenses in this survey) reflects the safety, efficacy, convenience and cost effectiveness of current-generation contact lenses.

The predominance of higher Dk materials used for OK fits versus non-OK fits can be attributed to the requirement to wear OK lenses overnight, in order to optimise corneal oxygen availability during sleep [27] and thus minimize ocular health complications [28].

That approximately two-thirds of OK lenses are replaced annually (compared with one-third of nonOK lenses) probably reflects the requirement to periodically alter lens design so as to maintain the desired corneal moulding effects [2].

\section{Conclusion}

$\mathrm{OK}$ is a niche contact lens fitting modality, which is employed by eye care practitioners in some European countries where there is a culture of rigid lens fitting supported by local laboratories capable of fabricating these lenses. Typically, OK lenses are fitted in high Dk materials on a planned replacement basis to younger patients, presumably for the primary purpose of arresting the progression of myopia.

\section{Acknowledgments}

The International Contact Lens Prescribing Survey Consortium: Philip B Morgan, United Kingdom; Nathan Efron, Australia; Craig A Woods, Australia; Jacinto Santodomingo-Rubido, Spain; Carmen Abesamis-Dichoso, The Philippines; Suresh Awasthi, Nepal; Joseph Barr, United States of America; Marion Beeler-Kaupke, Switzerland; Jitka Belikova, The Czech Republic; Vadim Belousov, Russia; Jolanta Bendoriene, Lithuania; Janet Casablanca, Puerto Rico; Aris Chandrinos, Greece; Prema Chane, India; Patrick Cheng, Hong Kong; JhoYan Chia, Malaysia; Byoung Sun Chu, South Korea; Edgar Dávila-Garcia, Puerto Rico; Nir Erdinest, Israel; Chi Shing Fan, AsiaPacific; Philip Fine, Israel; Peter Gierow, Sweden; José Manuel González-Méijome, Portugal; Martha Yanneth Gonzalez, United States of America; Hans-Jürgen Grein, Germany; Christina N Grupcheva, Bulgaria; Jörgen Gustafsson, Sweden; Magne Helland, Norway; Anna Yeo Chwee Hong, Singapore; Hreinn Ingi Hreinsson, Iceland; John Hsiao, Taiwan; Lee Kai Hung, Singapore; Motozumi Itoi, Japan; Ali Reza Jafari, Iran; Oskar Johansson, Sweden; Deborah Jones, Canada; Lyndon Jones, Canada; Razmig Knajian, United Arab Emirates; Jitka Krasnanska, The Czech Republic; Wanda Lam, New Zealand; Rafael S. Lemos, Brazil; Weronika Leszczyńska, Poland; Carla J Mack, United States of America; Patricia Magnelli, Argentina; Florence Malet, France; Edoardo Marani, Italy; Sebastian Marx, Germany; Nelson L. Merchan, Colombia; Giancarlo 
Montani, Italy; Jose L. Moroy; Mexico; Jason J. Nichols, United States of America; Alex Ong, Singapore; Guillermo Carrillo Orihuela, The Americas; Alice Pesinova, The Czech Republic; Geraint Phillips, New Zealand; Ricardo Pintor, Mexico; Athina Plakitsi, Greece; Heiko Pult, Germany; Polo Qi, China; Simona Radu, Romania; Jona Birna Ragnarsdóttir, Iceland; Hrvoje Raguž, Croatia; Ole Ravn, Denmark; Jeanette Romualdez-Oo, The Philippines; Svend-Erik Runberg, Denmark; Mirna Stabuc Silih, Slovenia; Danny Sim, Singapore; Leon Sze, Hong Kong; Kah-Ooi Tan, China; Panu Tast, Finland; Mario Teufl, Austria; Inga-Lill Thunholm-Henriksson, Sweden; Ioannis G Tranoudis, Greece; Marco van Beusekom, The Netherlands; Eef van der Worp, The Netherlands; Mihály Végh, Hungary; Edit Vodnyanszky, Hungary; Ann Elisabeth Ystenæs, Norway; and Veronica Ziziuchin, Moldova.

Declaration of interests: none.

\section{References}

[1] S.L. Ziff, Orthokeratology. 1, J Am Optom Assoc 39 (2) (1968) 143-147.

[2] P. Gifford, Orthokeratology. In: Efron N, ed. Contact Lens Practice. 3rd Edition. Edinburgh: Elsevier; 2018:296-304.

[3] J.J. Nichols, M.M. Marsich, M. Nguyen, J.T. Barr, M.A. Bullimore, Overnight orthokeratology, Optom Vis Sci 77 (5) (2000) 252-259.

[4] Y.M. Liu, P. Xie, The safety of orthokeratology - A systematic review, Eye Contact Lens 42 (1) (2016) 35-42.

[5] P. Cho P, Q. Tan, Myopia and orthokeratology for myopia control. Clin Exp Optom 2018 doi: 10.1111/cxo.12839.

[6] S.W. Cheung, M.V. Boost, P. Cho, Pre-treatment observation of axial elongation for evidence-based selection of children in Hong Kong for myopia control. Cont Lens Anterior Eye 2018 doi: 10.1016/j.clae.2018.10.006.

[7] P. Cho, S.W. Cheung SW. Protective role of orthokeratology in reducing risk of rapid axial elongation: A reanalysis of data from the ROMIO and TO-SEE Studies. Invest Ophthalmol Vis Sci 58 (3) (2017) 1411-1416.

[8] P. Cho, S.W. Cheung, Discontinuation of orthokeratology on eyeball elongation (DOEE). Cont Lens Anterior Eye 40 (2) (2017) 82-87.

[9] S.W. Cheung, P. Cho P, Long-term effect of orthokeratology on the anterior segment length. Cont Lens Anterior Eye 39 (4) (2016) 262-265.

[10] K.Y. Chan, S.W. Cheung SW, P. Cho P, Orthokeratology for slowing myopic progression in a pair of identical twins. Cont Lens Anterior Eye 37 (2) (2014) 116-119. 
[11] C. Chen, S.W. Cheung, P. Cho P, Myopia control using toric orthokeratology (TO-SEE study). Invest Ophthalmol Vis Sci 54 (10) (2013) 6510-6517.

[12] J. Charm, P. Cho P, High myopia-partial reduction ortho-k: a 2-year randomized study. Optom Vis Sci 90 (6) (2013) 530-539.

[14] J. Charm, P. Cho, High myopia-partial reduction orthokeratology (HM-PRO): study design. Cont Lens Anterior Eye 36 (4) (2013) 164-170.

[15] S.W. Cheung, Cho P, Validity of axial length measurements for monitoring myopic progression in orthokeratology. Invest Ophthalmol Vis Sci 54 (3) (2013) 1613-1615.

[16] P. Cho, S.W. Cheung, Retardation of myopia in Orthokeratology (ROMIO) study: a 2-year randomized clinical trial. Invest Ophthalmol Vis Sci 53 (11) (2012) 7077-7785.

[17] P. Chen, P. Cho P, Toric orthokeratology for high myopic and astigmatic subjects for myopic control, Clin Exp Optom 95 (1) (2012) 103-108.

[18] T.T. Lee, P. Cho P, Discontinuation of orthokeratology and myopic progression. Optom Vis Sci 87 (12) (2010) 1053-1056.

[19] P. Cho, S.W. Cheung, M. Edwards, The longitudinal orthokeratology research in children (LORIC) in Hong Kong: a pilot study on refractive changes and myopic control. Curr Eye Res 30 (1) (2005) 71-80.

[20] M.J. Lipson, M.M. Brooks, B.H. Koffler, The role of orthokeratology in myopia control: A review, Eye Contact Lens 44 (4) (2018) 224-230.

[21] P.B. Morgan, N. Efron, A decade of contact lens prescribing trends in the United Kingdom (1996 - 2005), Contact Lens Ant Eye 29 (2) (2006) 59-68.

[22] N. Efron, P.B. Morgan, C.A. Woods, The International Contact Lens Prescribing Survey Consortium, Survey of contact lens prescribing to infants, children and teenagers, Optom Vis Sci 88 (4) (2011) 461-468.

[23] N. Efron, P.B. Morgan, C.A. Woods, The international Contact Lens Prescribing Survey Consortium, International survey of rigid contact lens fitting, Optom Vis Sci 90 (2) (2013) 113-118.

[24] B.A. Holden, T.R. Fricke, D.A. Wilson, M. Jong, K.S. Naidoo, P. Sankaridurg, T.Y. Wong, T.J. Naduvilath, S. Resnikoff, Global prevalence of myopia and high myopia and temporal trends from 2000 through 2050, Ophthalmology 123(5) (2016) 1036-1042.

[25] J.S. Wolffsohn, A. Calossi, P. Cho, K. Gifford, L. Jones, M. Li, C. Lipener, N.S. Logan, F. Malet, S. Matos, J.M. Meijome, J.J. Nichols, J.B. Orr, J. Santodomingo-Rubido, T. Schaefer, N. Thite, E. van der Worp, M. Zvirgzdina, Global trends in myopia management attitudes and strategies in clinical practice, Cont Lens Anterior Eye 39 (2) (2016) 106-116. 
[26] I.G. Morgan, A.N. French, R.S. Ashby, X. Guo, X. Ding, M. He, K.A. Rose, The epidemics of myopia: Aetiology and prevention, Prog Retin Eye Res 62 (1) (2018) 134149.

[27] N.A. Brennan, Beyond flux: total corneal oxygen consumption as an index of corneal oxygenation during contact lens wear. Optom Vis Sci 82 (6) (2005) 467-472.

[28] N. Efron, Contact Lens Complications, $4^{\text {th }}$ ed. Edinburgh: Elsevier; 2019. 


\section{Figure Legends}

Fig. 1. Proportion all contact lenses fitted as orthokeratology lenses in 45 countries between 2004 and 2017. Error bars represent the upper 95\% confidence limit. AE United Arab Emirates; AR Argentina; AT Austria, AU Australia; BE Belgium, BG Bulgaria; BR Brazil; CA Canada; CH Switzerland; CN China; CZ Czech Republic; DE Germany; DK Denmark; EG Egypt; ES Spain; FR France; GR Greece; HK Hong Kong; HU Hungary; ID Indonesia; IL Israel; IR Iran, IT Italy; JO Jordan; JP Japan; KR South Korea; LT Lithuania; MX Mexico, MY Malaysia; NL Netherlands; NO Norway; NP Nepal; NZ New Zealand; PH Philippines; PR Puerto Rico; PT Portugal; RO Romania; RU Russia; SE Sweden; SG Singapore; SI Slovenia; TW Taiwan; UK United Kingdom; US United States; ZA South Africa.

Fig. 2. 'Heat map' indicating the extent of orthokeratology lens fitting in different countries around the world. Note the logarithmic colour-coded scale. Nations shown in white (no colour) were not surveyed.

Fig. 3. Trend in the proportion all contact lenses fitted as orthokeratology lenses in 45 countries between 2004 and 2017. Error bars represent the 95\% confidence limit.

Fig. 4. Age categories of persons fitted with contact lenses for orthokeratology (OK), and for persons fitted with all other lens types (Non-OK), in 45 countries between 2004 and 2017. 


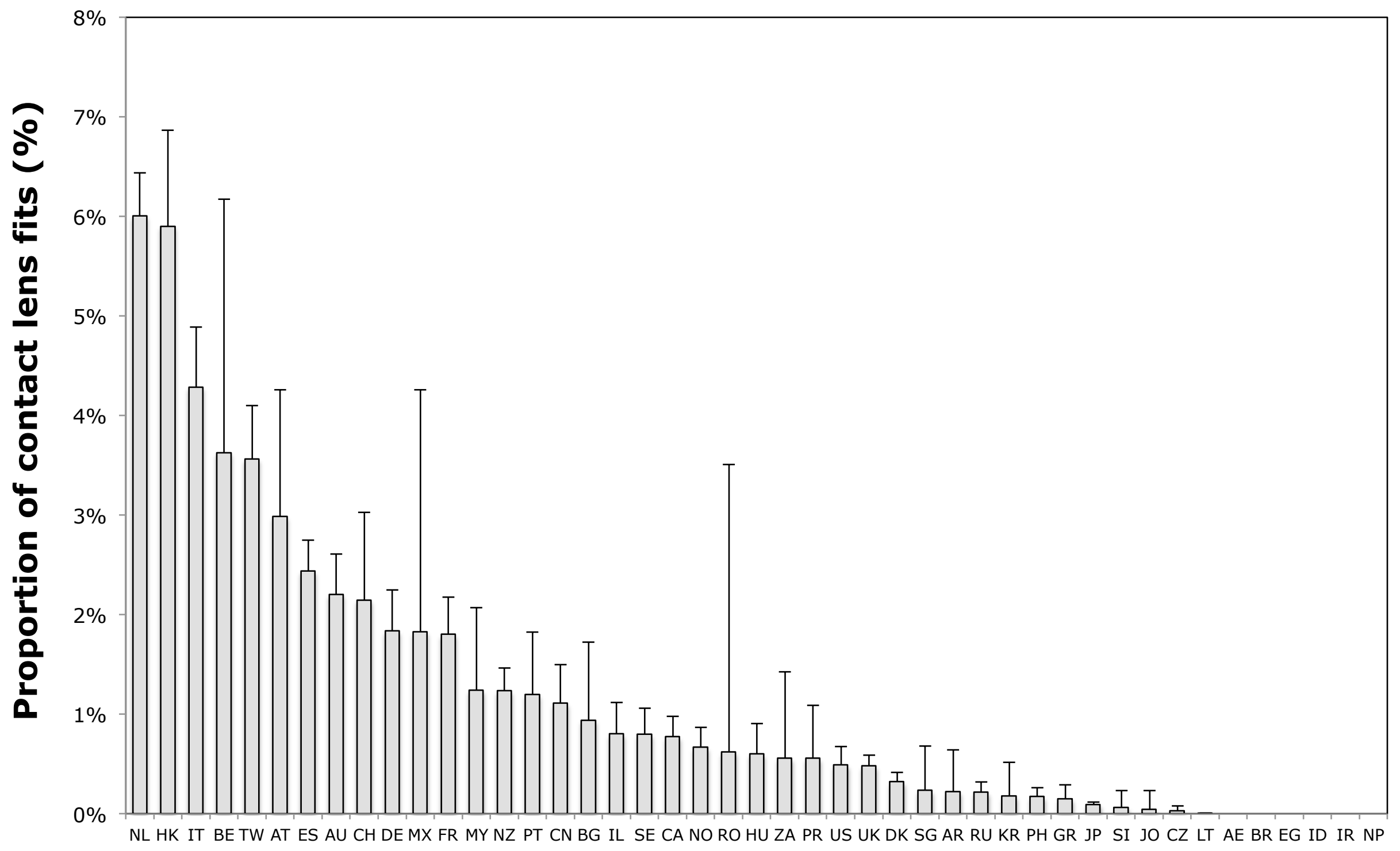




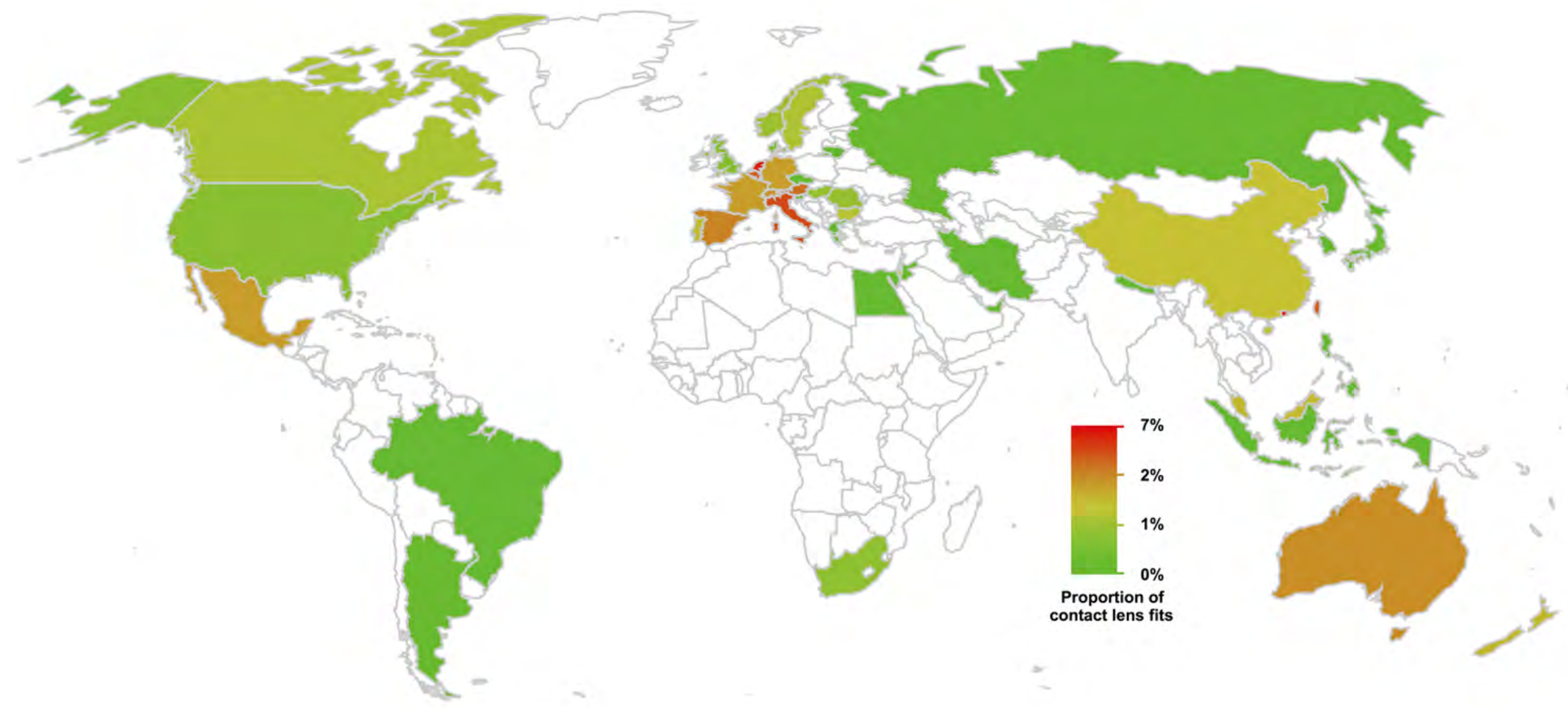




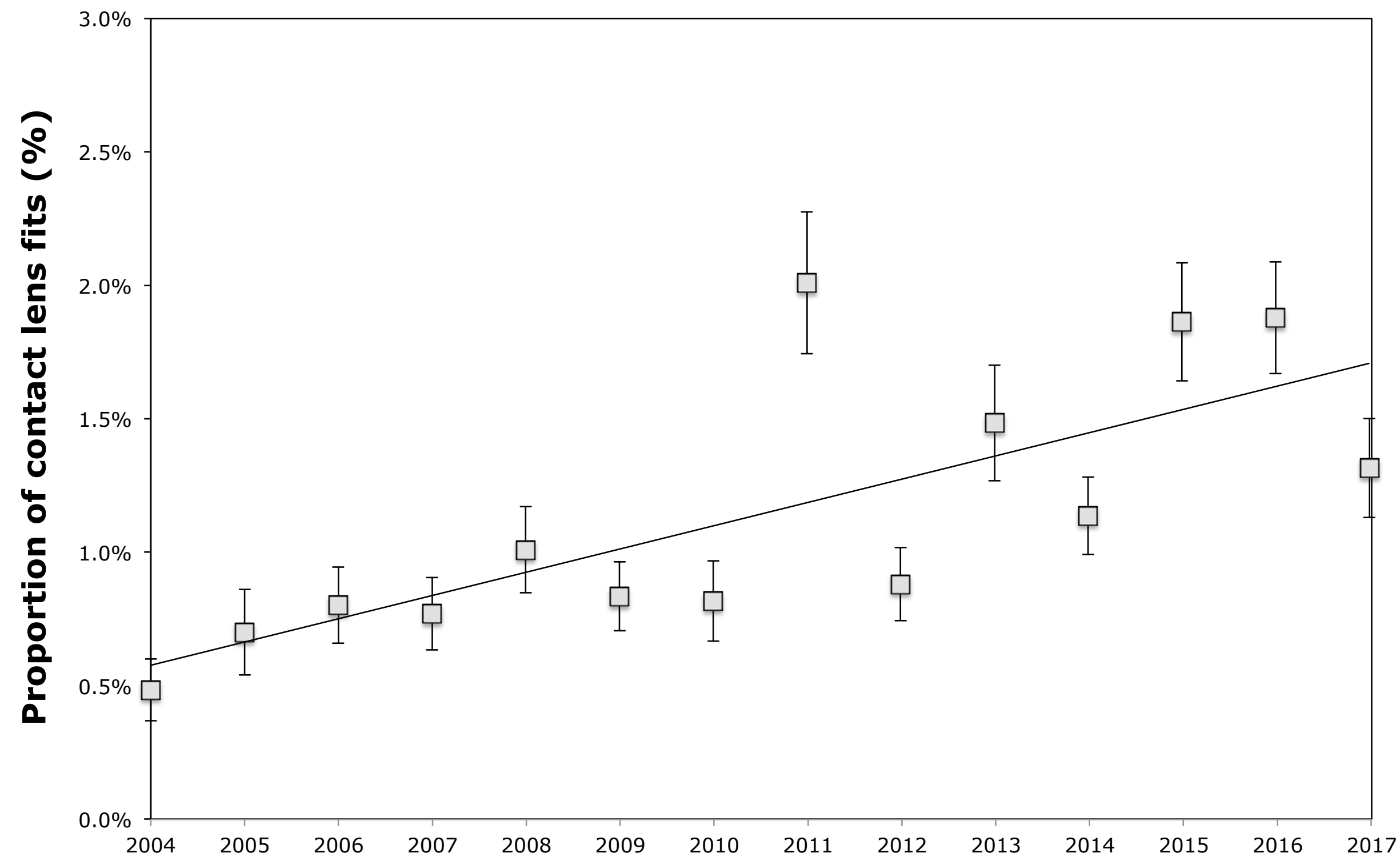




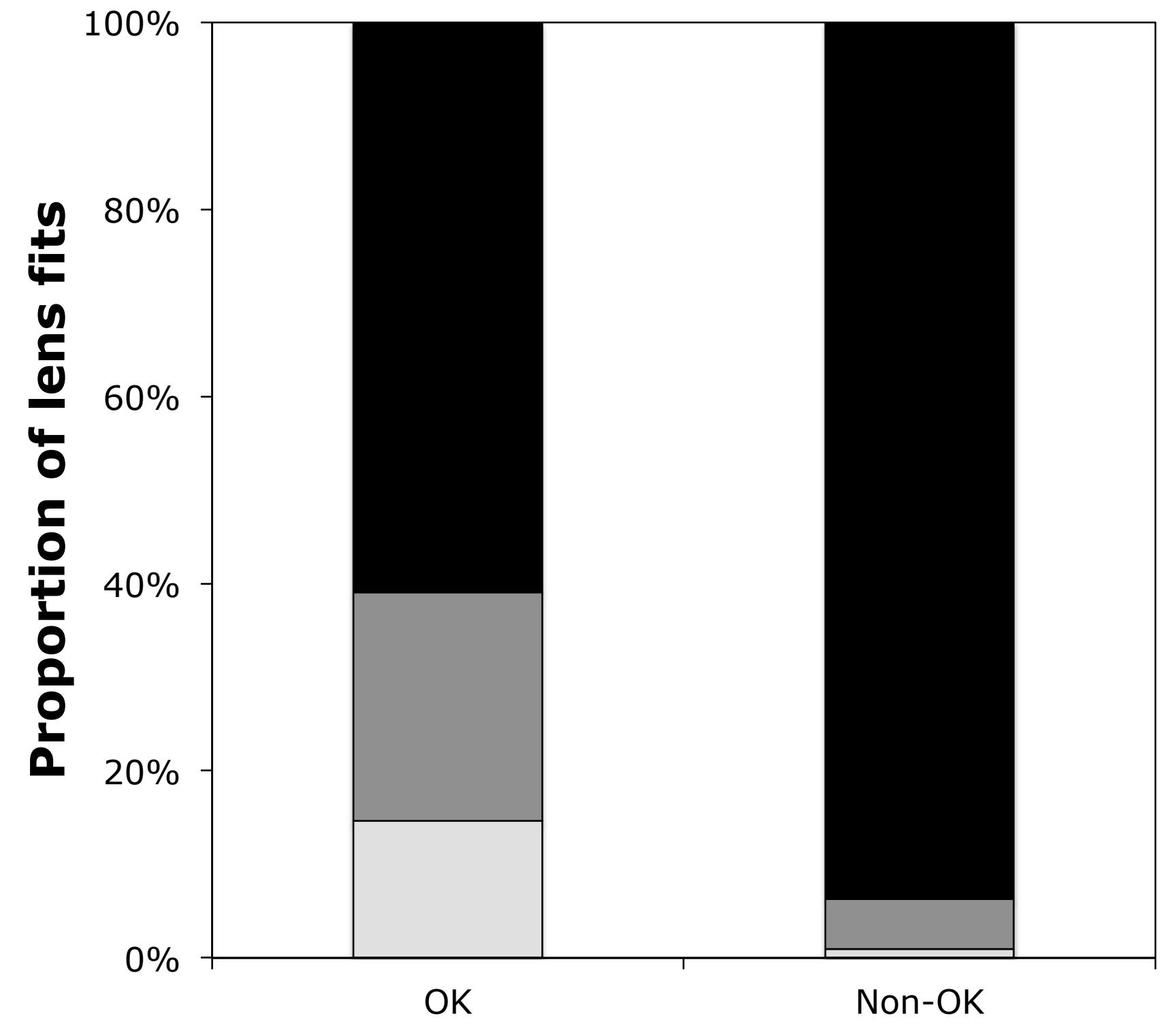

- Adults ( $\geq 18 \mathrm{yr}$ )

$\square$ Teenagers (13-17yr)

$\square$ Children (6-12yr)

$\square$ Infants (0-5yr) 